\title{
Emotion, Well-Being, and Resilience-Theoretical Perspectives and Practical Applications
}

\author{
Editors Dr. Rabindra Kumar Pradhan \& Dr. Updesh Kumar, Publisher: Apple \\ Academic Press, 2021. Pages: 532, ISBN (hardcover): 9781771888905, ISBN (eBook): \\ 9781003057802
}

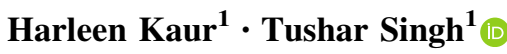

Received: 15 June 2021 / Accepted: 24 January 2022/Published online: 28 February 2022

(C) National Academy of Psychology (NAOP) India 2022

\begin{abstract}
Resources are required to minimize the daily threats in life; thus, discussions about the role of emotions, well-being, and resilience become imperative for human survival. The book reviewed here attempts to highlight the associations among human well-being, emotions, and resilience. The book showcases resilience as a conceptually and empirically robust construct useful for research and practice, and takes a comprehensive endeavour to provide the importance of resilience in various contexts by presenting suitable empirical evidences and applications. The book is deeply rooted in positive psychology and would be meaningful for scholars, leaders of organizations, and health professionals working in the areas of counselling, well-being, and community development.
\end{abstract}

Keywords Resilience · Emotions · Well-being ·

Human survival · Positive psychology

Living in the present times is supplemented by fierce competition. Today, the world is witnessing changes in the dynamics of lifestyle due to globalization (Baek et al., 2009), automation (Laamarti \& El Saddik, 2017), technologies (Hanafizadeh et al., 2017), and modernization (Janus et al., 1996). The outbreak of the coronavirus pandemic raged the fuel to this developing narrative (Ylldiz, 2020). Consequently, social apathy, violence, vandalism, and aggression rushing in humans accelerated the survival threat around the globe. Given the destabilized life in

Tushar Singh

tusharsinghalld@gmail.com

1 Department of Psychology, Banaras Hindu University, Varanasi, U. P 221005, India community and society that we are dealing with at present, it is likely that we must continuously adjust ourselves in this harsh environment. Therefore, it is indispensable for us to rejuvenate our available resources and learn how to build the new ones.

Keeping in view the 'resources' required to minimize the threats in different contexts daily, discussion about the role of emotions, well-being, and resilience becomes imperative for human survival. There exists sufficient literature to strengthen our understanding of emotions, how to manage them for our overall well-being, and how it can help us build resilience. The present book entitled 'Emotion Well-Being and Resilience-Theoretical perspectives and Practical Applications' is one such volume that underlies the association of well-being with emotions, along with the resilience, and subsequently, present one of the most important requirements of the current world. Contributed by more than sixty scholars from across six continents, this volume documents the concepts, research, and practices underlying resilience, which refers to qualities an individual needs to face adversity (p.15), and its association with emotions and well-being.

Although a range of literature is available on issues concerning emotions; well-being and resilience, the majority of them discusses only the conceptual and understandable consequences of unmanageable emotions, poor well-being (Hendriks et al., 2021) and presents only the detrimental effects of these variables (Connor \& Zhang, 2006). The present volume, however, is rooted deeply in positive psychological approach and provides a complete picture starting from the genesis of stress to the capacity of resilience escalated to the on-going advancements in the domain of emotional regulation. It brings forth the recent development of positive psychology all together with a comprehensive understanding of theoretical underpinnings 
along with empirical evidence, for example, the concept of cherishing under psychological well-being is discussed in the volume. The volume adopts a cross-cultural multifactorial approach wherein, the context of resilience is presented embodied from different cultures and practised throughout the globe, while simultaneously highlighting every other factor which can or should contribute to building resilience. For example, the book throws light on the relation between resilience and well-being in context of other geographical locations such as India, Czech Republic, South Africa, Peru, the USA, and South Korea. The handbook is comprised of 31 chapters divided into four sections each of which is thematically arranged. Every section in the volume begins with presenting the conceptual underpinning of resilience in that particular context, progresses to discuss the empirical evidence of the same, and ends while offering the applications and implications for the same context to the readers.

Clinical and Counselling Context: The first section incorporates twelve chapters to provide a comprehensive understanding of emotions, well-being, and resilience in the clinical and counselling context. As Southwick et al. (2014) highlighted the association of stress and resilience with coping, or as intentionally posed self-injury described by Nock (2010), this section provides the understanding of emotions and resilience with the meaning of life and selfdestructive behaviour. The section also discusses the association between social support and emotional regulation and describes the association of stress with perceived hope, satisfaction of cancer patients, correlates of self-efficacy and happiness, chronic illness, and suicide risk along with discussing the therapeutic implications of emotion regulation.

Home, School, Community, and Sports Environment: Section two includes six chapters that discuss the constructs of resilience in the environment outside the context of clinical and counselling settings and presents a thorough understanding of resilience and emotions in the home, school, community, and sports settings. Here, the association of resilience is addressed with family, successful ageing, studentship, and teaching. Moreover, the role of resilience for place identity in the community, as well as how it is a boon for the athletes' well-being is also highlighted.

Work and Organizational Context: The domain of resilience in the context of workplace and organization is described in the third section containing seven chapters. Here, the link of resilience is seen with positive emotions, leadership, health, balance, psychological capital and life satisfaction, work scale, techno-stress, as well as with employee's well-being.

Extreme Environment: The fourth section, having six chapters, illustrates the impact of resilience in extreme environments. Explaining the association of resilience with psychological flexibility, the ability which emerges as the process of adaptation, reconfiguration, shift, and balance (Kashdan \& Rottenberg, 2010) is discussed in the military context, underlying terrorist threat, and also presents the transformation of humans during post-traumatic growth. Moreover, the section also explores the role of social support and resilience in adventure programming.

Strength and limitations of the book: The book attempts to describe the utility of resilience not only at the microlevel (individual, family, and community) but also at the macro-level (organizations, terrorism, and military) environments. It presents the reader with the understanding and identification of the concept of resilience in a particular context. In doing so, suitable empirical evidences are presented supplemented with the appropriate applications in that context of resilience. The volume takes a comprehensive endeavour to present both sides of the coin. Here, the factors predicting harmful key risks are supplemented with the best contributors of growth; consequently, this serves the complete picture of resilience. Moreover, it expands the horizon of resilience in some foreseen domains, as discussed in work put forward by Glass \& Mayers (2001) on adventure programming, in holistic perception of social support during post-traumatic growth, and in overlapping and confusion of post-traumatic growth. The volume also highlights the role of resilience in shared identity and belongingness in terrorism, autonomy, the cognitive and emotional domain of psychological flexibility, especially in military personals. Emotional well-being, workspace and employees' performances, techno-stress, insecurity, overload, uncertainty (Tarafdar et al., 2007), and turnover intention (Kim \& Stoner, 2008) are discussed as well. This volume also presents resilience at work (RAW) scale (Winwood et al., 2013) in the Indian settings and adds five new dimensions to the pre-existing seven dimensions of the RAW scale. It also discusses novel concepts such as cross-over effects (Westman, 2001) in dual working couples, contemporary psycho-social hazards, positive organizational behaviour, mental training of athletes, place identity in community, demographics of teachers, chronic illness in the paradigm of health psychology. Resilience could also be linked to disability and happiness though they are poles apart, patient satisfaction with doctor consultation, feelings, hope, and harmony, 'cherishing' with six sub-components of psychological well-being, challenges of intersecting identities are also underlined when self-destructive behaviour is linked with resilience.

The volume also places emphasis on both positive and negative emotions. It interchangeably used words like 'optimism, enthusiasm, passion, engagement, and selfmotivation' to describe positive emotions. On the contrary, 
negative emotions are attributed as 'negativism, pessimism, helplessness, hopelessness'. Such notations give a clear and concise differentiation between the two felt emotions. Overall, this book conceptually proposes the need for assessment, tools, and resilience training, providing a multifactorial approach to understand the concept of resilience wherein the negative factors could be minimized and personal growth is maximized.

Though the contributors have tried their best to illustrate every domain and predictor of resilience, they have outlined the working on 'self' the most in this volume. This also serves as limitation of the book, since the volume makes the reader inquisitive to dwell for understanding the 'self' thoroughly; the reader further expects going through the underpinnings of 'self-awareness' with respect to resilience, which is being missed in this volume. Learning this, it is expected that the next volume in this series would provide a holistic picture by giving a better overview of self, awareness, and evaluation.

Take-away from the volume: The volume asserts that when one attains the highest form of resilience they are capable of strengthening others. Practically, it is possible in a workspace wherein a leader can train his/her subordinate by resilience-building programmes that aim to polish individuals' performance while enhancing their productivity for completing stressful tasks. Likewise, parents can be the medium in the home environment. This programme can be included in the curriculum for the school settings. Such training can be boon to upcoming generations who would be developing sustainably. Building 'bouncing back capacity' can make them face every harsh turn while simultaneously complimenting their personal growth. Similarly, to stabilize the staff working in high-risk professions, the multifactorial model of resilience may serve as a sword to fight unprecedented situations.

This volume would be meaningful for scholars, leaders of organizations, and health professionals working in the areas of counselling, well-being, and community development. It is a valuable resource to learn about the positive and the negative sides of resilience. Importantly, it clarifies resilience as a conceptually and empirically robust construct useful for research and practice. Moreover, this volume enables us to implement the reading in practice to understand the risk factors and eventually inculcate the strength of 'bouncing back to life'.

Authors' contributions All the authors contributed equally in the preparation of this review.

Funding The authors received no specific funding for this work.

Availability of data and material Not applicable.
Code availability Not applicable.

\section{Declarations}

Conflict of interest We have no known conflict of interest to disclose.

Ethics approval Not applicable.

Consent to participate Not applicable.

Consent for publication Not applicable.

\section{References}

Baek, J., Cho, Y., \& Koo, W. W. (2009). The environmental consequences of globalization: A country-specific time-series analysis. Ecological Economics, 68(8-9), 2255-2264.

Connor, K. M., \& Zhang, W. (2006). Resilience: Determinants, measurement, and treatment responsiveness. CNS Spectrums, 11(S12), 5-12.

Glass, J. S., \& Myers, J. E. (2001). Combining the old and the new to help adolescents: Individual psychology and adventure-based counseling. Journal of Mental Health Counseling, 23(2), 104-114.

Hanafizadeh, P., Ghandchi, S., \& Asgarimehr, M. (2017). Impact of information technology on lifestyle: A literature review and classification. International Journal of Virtual Communities and Social Networking (IJVCSN), 9(2), 1-23.

Hendriks, T., Schotanus-Dijkstra, M., Graafsma, T., Bohlmeijer, E., \& de Jong, J. (2021). Positive emotions as a potential mediator of a multi-component positive psychology intervention aimed at increasing mental well-being and resilience. International Journal of Applied Positive Psychology, 6, 1-21.

Janus, E. D., Postiglione, A., Singh, R. B., \& Lewis, B. (1996). The modernization of Asia: Implications for coronary heart disease. Circulation, 94(11), 2671-2673.

Kashdan, T. B., \& Rottenberg, J. (2010). Psychological flexibility as a fundamental aspect of health. Clinical Psychology Review, 30(7), 865-878.

Kim, H., \& Stoner, M. (2008). Burnout and turnover intention among social workers: Effects of role stress, job autonomy and social support. Administration in Social Work, 32(3), 5-25.

Laamarti, F., \& El Saddik, A. (2017). Home automation serving a healthier lifestyle. In 2017 IEEE International Symposium on Medical Measurements and Applications (MeMeA) (pp. 39-44). IEEE.

Nock, M. K. (2010). Self-injury. Annual Review of Clinical Psychology, 6, 339-363.

Southwick, S. M., Bonanno, G. A., Masten, A. S., PanterBrick, C., \& Yehuda, R. (2014). Resilience definitions, theory, and challenges: Interdisciplinary perspectives. European Journal of Psychotraumatology, 5(1), 25338.

Tarafdar, M., Tu, Q., Ragu-Nathan, B. S., \& Ragu-Nathan, T. S. (2007). The impact of technostress on role stress and productivity. Journal of Management Information Systems, 24(1), 301-328.

Westman, M. (2001). Stress and strain crossover. Human Relations, 54(6), 717-751.

Winwood, P. C., Colon, R., \& McEwen, K. (2013). A practical measure of workplace resilience. Journal of Occupational and Environmental Medicine, 55(10), 1205-1212. https://doi.org/ 10.1097/jom.0b013e3182a2a60a 
Y1ldız, E. (2020). What can be said about lifestyle and psychosocial issues during the coronavirus disease pandemic? first impressions. Perspectives in Psychiatric Care.
Publisher's Note Springer Nature remains neutral with regard to jurisdictional claims in published maps and institutional affiliations. 